\title{
Configuring Health Care for Systematic Behavioral Screening and Intervention
}

\author{
Richard L. Brown, MD, MPH
}

\begin{abstract}
The United States Preventive Services Task Force recommends universal screening and intervention for tobacco use, excessive drinking, and depression. These services improve health outcomes, decrease health care costs, enhance public safety, and generate substantial return on investment. Given the prevalence rates of these behavioral conditions and the time necessary for evidence-based interventions, it will be challenging to integrate behavioral screening and intervention (BSI) into busy health care settings. Therefore, consistent with the principles of the medical home and the chronic care model, the health care team must be expanded to systematically provide BSI. A 2-tiered, stepped-care model is proposed. The first tier of services-consisting of assessment, intervention, and follow-up services-would address most mild-to-moderate behavioral risks or conditions. The second tier would include various specialty-based resources, which would be conserved for patients with greatest need and potential to benefit. With slight enhancement of their training, health educators would be excellent candidates to serve as cost-efficient providers of first-tier services. The proposed model would help the United States realize improved health outcomes and cost savings as health care benefits are expanded to a greater proportion of its population. (Population Health Management 2011;14:299-305)
\end{abstract}

\section{The Imperative for Systematic Behavioral Screening and Intervention (BSI)}

B EHAVIORS SUCH AS TOBACCO USE, excessive drinking, drug use, poor diet, and physical inactivity are responsible for $40 \%$ of deaths, most chronic diseases, and disability, and $\$ 539$ billion in costs for health care and productivity loss in the United States. ${ }^{1-6}$ Depression exerts additional burden through poor quality of life, productivity loss, poor selfmanagement of chronic illness, and suicide, and carries an additional $\$ 82$ billion in costs. ${ }^{7,8}$ A robust literature shows that systematically screening all patients for tobacco use, ${ }^{9}$ excessive drinking, ${ }^{10}$ and depression ${ }^{7}$ improves health outcomes. Tobacco cessation nearly pays for itself over several years through reduced health care utilization, and employers experience additional benefits through lower absenteeism and higher productivity. ${ }^{9}$ Depression services generate a 3-to1 return on investment over 2 years. ${ }^{11}$ Alcohol services reduce hospitalizations, emergency department utilization, car crashes, and arrests, and generate a 4-to- 1 or greater return on investment in 1 year. ${ }^{12-14}$ Under health care reform, payers are required to reimburse for these services without out-ofpocket expenditures by patients.
Different terminology has evolved for screening, intervention, and referral for various behavioral issues. The 5 A's-ask, advise, assess, assist, and arrange-apply to tobacco use. ${ }^{9}$ SBIRT (screening, brief intervention, and referral to treatment) pertains to alcohol and drug use. ${ }^{15}$ Collaborative care consists of a team approach to delivering the assessment, intervention, referral, and follow-up services recommended for patients who screen positive for depression. $^{16}$

For all behavioral issues, BSI begins with patients answering 1 to 4 screening questions on each behavioral issue. Patients with positive screens undergo further assessment.

For patients who have not already decided to change their tobacco use, excessive drinking, or drug use, initial services are directed at eliciting desire to change. They range from brief motivational interventions, which can take as little as a few minutes, ${ }^{17}$ to extended motivational interventions over several encounters. ${ }^{18}$ Some patients who are committed to change need no support, but for those who do, typical services, based on relapse prevention, ${ }^{19}$ help them design behavior change plans, execute them, and refine them over time to maximize their success. For patients with severe behavioral disorders, the change plan may include referral to

Department of Family Medicine, School of Medicine and Public Health, University of Wisconsin, Madison, Wisconsin. 
self-help, group support, pharmacotherapy, and/or other specialized treatment. For all patients, follow-up is recommended to assess for progress and needs.

For patients who screen positive for depression, collaborative care also starts with assessment. Potentially suicidal patients are referred immediately for specialized assessment and triage. Patients with major depression receive education and referrals for pharmacotherapy and/or psychotherapy. Patients are supported in adhering to treatment plans. Regular reassessments with standardized assessment tools guide clinicians in modifying treatment as necessary. ${ }^{16}$

Collaborative care can be supplemented with a behavioral activation intervention, which promotes behaviors that decrease depression symptoms such as engaging in exercise, healthier nutrition, social activities, fun, sleep hygiene, and relaxation. ${ }^{20}$ Behavioral activation interventions can avert major depression for patients with minor depression. ${ }^{21} \mathrm{~Pa}$ tients with major depression also benefit from behavioral activation interventions. ${ }^{22}$ In fact, it is important that treatment for major depression includes a behavioral modality, as such modalities protect better against relapse than pharmacotherapy, ${ }^{23}$ and pharmacotherapy alone is ineffective for many patients with mild-to-moderate depression. ${ }^{24}$

Because of ample documentation of effectiveness, tobacco, alcohol, and depression screening have received Grade A or B ratings from the US Preventive Services Task Force. ${ }^{25}$ The National Commission on Prevention Priorities ranks tobacco and alcohol screening third and fourth in effectiveness and return on investment-just behind aspirin for cardiovascular prevention and immunizations for children, and ahead of screening for hypertension, dyslipidemia, diabetes, and all forms of cancer. Depression is ranked eighteenth of 25 preventive services and would have been ranked higher had improvements in workplace productivity been considered. ${ }^{26}$ A growing literature suggests that screening and intervention for drug use is effective. ${ }^{15,27-31}$

Unfortunately, although the growing prevalence of overweight and obesity is of great concern, the effectiveness of screening and intervention for these behavioral issues is limited. Many individuals enhance their fruit and vegetable intake after brief educational feedback. ${ }^{32} \mathrm{~A}$ brief motivational intervention can elicit modest weight loss, ${ }^{33}$ but whether a more extended intervention can increase and sustain weight loss is not known. Interventions increase exercise by healthy individuals but only with ongoing support. ${ }^{34}$ The US Preventive Services Task Force recommends dietary interventions for patients with cardiovascular risk factors but does not recommend interventions on diet or physical activity for healthy individuals. ${ }^{35,36}$

In summary, the effectiveness and return on investment of BSI is well documented for tobacco use, excessive drinking, and depression. Because of the growing evidence of effectiveness of brief interventions for drug use and the importance of reducing drug use for public health and public safety, it would be reasonable to screen and intervene for drug use along with alcohol use. Screening and intervention has a limited role for healthy patients with unhealthy diets and inadequate physical activity. Perhaps a reasonable level of services for nutrition and exercise would consist of screening, brief feedback, recommendation, and referral for interested patients. The time has come to systematically deliver BSI in general medical settings.

\section{Designing Systematic BSI}

Systems to deliver BSI to the 250 million adults and teens across the United States must be designed to maintain patient flow through busy health care settings, to minimize cost, and to maximize return on investment. Cost-efficiency requires that all staff operate at their maximum skill level.

In primary care settings, initial screening for all behavioral issues is most easily conducted in writing, though some patients require personal assistance to overcome communication barriers. Paper questionnaires are used most commonly, though electronic devices could enhance the efficiency of data entry and record keeping. Receptionists can initiate screening by distributing questionnaires when patients register. Staff members who take vital signs can quickly review questionnaire responses, measure height and weight, and add body mass index to the screen.

In emergency departments and on hospital inpatient units, similar screening would be more difficult to coordinate because of larger numbers of staff. Thus, a preferred option might be for the staff who deliver assessment, intervention, and referral services to approach patients, explain their role, and conduct screening, either in writing or orally. Such staff should be advised which patients are too ill, uncomfortable, or mentally compromised to participate.

\section{Need to Expand the Health Care Team}

A key question in BSI system design is who will conduct screening in emergency departments and hospitals and respond to positive screens in all settings. To answer this question, one important consideration is the prevalence of behavioral risks and conditions. US population prevalence data are shown in Figure 1. Prevalence for some behaviors and conditions is likely higher in clinical populations. Although prevalence varies by location, over $90 \%$ of most patient populations can be expected to have a positive screen for at least one unhealthy behavior or behavioral condition.

Another consideration is how much time it would take to address each behavioral issue for which a patient screens positive. Data are scarce on the time required to address excessive drinking and depression effectively. Optimal tobacco outcomes are obtained with a total of 0.5 to 5 hours of one-on-one counseling over more than 8 visits. ${ }^{9}$ If generating reimbursement for BSI is important to finance service delivery, then minimum time requirements must be considered, as shown in Table 1. Reimbursement notwithstanding, Table 2 shows a conservative estimate of the time required-

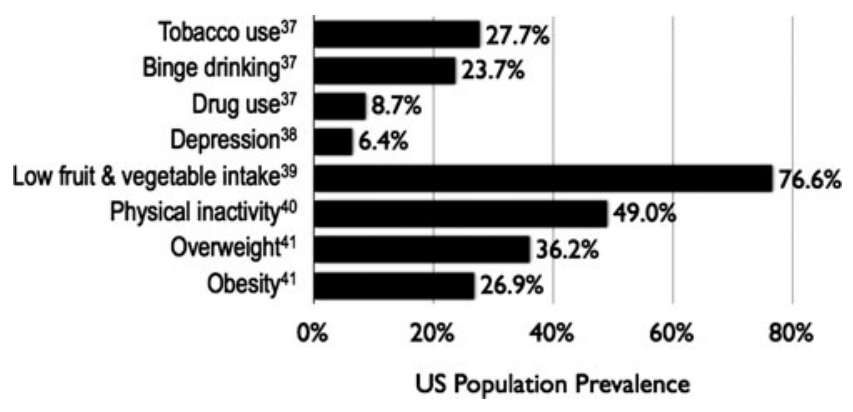

FIG. 1. Prevalence of risky behaviors and behavioral conditions among US adults. 
Table 1. Billing Time Requirements for Behavioral SCREENING AND INTERVENTION

\begin{tabular}{|c|c|c|}
\hline Billing Code & Service & $\begin{array}{c}\text { Time } \\
\text { Specification }\end{array}$ \\
\hline СРТ 99406 & $\begin{array}{l}\text { Tobacco screening and/ } \\
\text { or intervention }\end{array}$ & $\begin{array}{l}>3 \text { to } 10 \\
\text { minutes }\end{array}$ \\
\hline СРТ 99407 & $\begin{array}{l}\text { Tobacco screening and/ } \\
\text { or intervention }\end{array}$ & $>10$ minutes \\
\hline СРТ 99408 & $\begin{array}{l}\text { Alcohol and/or drug } \\
\text { screening and/or } \\
\text { intervention }\end{array}$ & $\begin{array}{l}15 \text { to } 30 \\
\text { minutes }\end{array}$ \\
\hline СРТ 99409 & $\begin{array}{l}\text { Alcohol and/or drug } \\
\text { screening and/or } \\
\text { intervention }\end{array}$ & $>30$ minutes \\
\hline HCPCS H0049 & $\begin{array}{l}\text { Alcohol and/or drug } \\
\text { screening }\end{array}$ & [none] \\
\hline HCPCS H0050 & $\begin{array}{l}\text { Alcohol and/or drug } \\
\text { intervention }\end{array}$ & $\geq 15$ minutes \\
\hline
\end{tabular}

CPT, Common Procedural Terminology; ${ }^{42}$ HCPCS, Healthcare Common Procedures Coding System. ${ }^{43}$

over 14 minutes-for an initial BSI session that addresses each positive screen. Additional time would be required for follow-up visits.

A common approach to implementing BSI involves asking current clinical staff to add BSI to their current responsibilities. This approach usually fails because of extant clinical and administrative demands, the high number of patients with positive screens, and the substantial time necessary to deliver effective interventions. Indeed, to deliver all recommended prevention services to an average-size patient panel, primary care providers would have to spend more than 7 hours every day. ${ }^{44}$ Consistent with the principles of the medical home ${ }^{45}$ and the chronic care model as adopted for prevention, ${ }^{46}$ the health care team must be expanded if behavioral risk factors and conditions are to be systematically addressed.

Table 2. Estimated Time Requirements FOR THE INITIAL BSI ENCOUNTER

\begin{tabular}{lccc}
\hline Behavioral issue & Prevalence & Time/Patient & Total Time \\
\hline Tobacco & 0.277 & 10 & 2.8 \\
Binge drinking & 0.237 & 10 & 2.4 \\
Drug use & 0.087 & 10 & 0.9 \\
Depression & 0.064 & 20 & 1.3 \\
Diet & 0.766 & 3 & 2.3 \\
Activity & 0.490 & 3 & 1.5 \\
Overweight & 0.362 & 5 & 1.8 \\
Obesity & 0.269 & 5 & 1.3 \\
Grand total & & & 14.2 \\
\hline
\end{tabular}

Times are shown in minutes. "Prevalence" is taken from Figure 1. "Time/patient" is the conservatively estimated intervention time for each patient who screens positive for each behavioral condition. "Total time" is derived by multiplying "Prevalence" and "Time/ patient." "Grand total" is the conservatively estimated intervention time for an average American adult across all behavioral conditions. Estimates pertain only to initial visits. Additional time is required for follow-up visits.

BSI, behavioral screening and intervention.

\section{How to Expand the Health Care Team}

One way to expand the team would be to hire multiple individuals who each have specialty training in a behavioral area. This solution would be untenable for most settings, which could neither fully utilize nor house a full-time tobacco cessation specialist, alcohol/drug counselor, mental health professional, dietitian, and exercise coach. Even in large settings where such staff could be absorbed, the many patients who screen positive in multiple behavioral areas would find it inconvenient and impersonal to meet with multiple staff. From a systems perspective, having 1 individual respond to positive screens in all areas would maximize economy of scale and time and space efficiency.

Furthermore, for patients with multiple positive screens, a single professional with appropriate knowledge and skill is more likely to succeed than multiple professionals with special expertise in a single behavioral realm. One reason is that a single interventionist will establish more rapport and gain a deeper understanding of patients' lives. Also, whereas US health care professionals typically have regarded each of these behavioral issues as a specialized area, for most patients they are highly interrelated. For example, drinking, drug use, or depression often engenders more tobacco use. Attempts to quit smoking generate more food consumption. Disinhibition from drinking can lead to lapses in attempts to change other behaviors. Common life stresses aggravate all behavioral issues. Thus, care from a single generalist behavioral practitioner would address the interplay among patients' behavioral issues better than fragmented care from multiple behavioral specialists.

Another consideration is that most individuals have behavioral issues of mild-to-moderate acuity and severity and may not need specialized care. The skills to manage behavioral issues of lower severity (ie, administering brief interventions, basic motivational interviewing, behavioral activation, relapse prevention, and basic case management) are not as complex or as dependent on clinical judgment as psychotherapies. These basic behavior change techniques can be guided in part by protocol and taught to appropriately selected baccalaureate-level individuals. For example, in Substance Abuse and Mental Health Services Administration-funded, state-based SBIRT programs, which have elicited substantial reductions in drinking and drug use in dozens of sites, most services have been delivered by paraprofessionals who were guided by paper or electronic protocols. ${ }^{15}$ Studies document that practitioners and students of diverse professions can help patients change a variety of risky and unhealthy behaviors through motivational interviewing. ${ }^{47}$ A pilot study found that protocol-guided behavioral activation and collaborative care delivered by baccalaureate-level paraprofessionals elicited high patient and provider satisfaction and 55\% declines in depressive symptoms over several weeks (K.R. Breidenbach, M.D. Croyle, L.A. Saunders, T.E. Woods, R.L.B., unpublished data, 2011).

We therefore propose a 2-tiered system for delivering BSI, as shown in Table 3 . In the first tier, a single individual serves as the initial responder to patients with positive screens, administers validated assessment questionnaires, and delivers brief feedback, brief and extended motivational interventions, and behavioral activation interventions for 
Table 3. A Two-Tiered Model for Systematically Delivering Behavioral Screening and Intervention

\begin{tabular}{|c|c|c|c|c|c|c|}
\hline Tier & Acuity and Severity & Tobacco & Alcohol and Drugs & Diet & Exercise & Depression \\
\hline \multirow[t]{3}{*}{1} & Unknown & \multicolumn{5}{|c|}{ Conduct assessment by administering validated questionnaires } \\
\hline & Low & BI, MI and Ref & BI, MI, RP & BFRR & BFRR & BA \\
\hline & High & & Ref & Ref & Ref & BA and Ref \\
\hline 2 & High & Ph and GS & $\mathrm{Ph}, \mathrm{GS}$ and ST & GS and ST & GS & $\mathrm{Ph}, \mathrm{Ps}$, and ST \\
\hline
\end{tabular}

BA, behavior activation; BFRR, brief feedback, recommendation and referral; BI, brief intervention; GS, group support; MI, motivational interviewing; Ph, Pharmacotherapy; Ps, Psychotherapy; Ref, Referral to Tier 2 services; RP, relapse prevention; ST, specialized treatment.

patients with behaviors and behavioral conditions of low acuity and severity. For behavioral conditions of higher acuity or severity, such as addiction or major depression, the first tier provider refers patients to a variety of specialized referral resources.

\section{What Profession Is Best Suited to be a First-Tier BSI Provider?}

Currently no health profession equips its practitioners with all the knowledge and skills to fulfill this first-tier role across all behavioral issues. Perhaps the most cost-efficient professionals who come closest are health educators, who have already been recognized for the important roles they could serve in medical homes. ${ }^{48}$ Nationally established competencies for health educators include assessing individual and community needs for health education; planning, implementing, evaluating and administering interventions and programs; serving as a health education resource; and advocating for health and health education. ${ }^{49}$ Health educator trainees typically complete coursework on epidemiology, biostatistics, research methods, program development and evaluation, quality improvement, disease prevention and control, health behaviors, sexual health, nutrition, mental health, substances and society, injury and violence, and aging. Training programs that are enhanced with a special BSI track could equip health educators with the additional knowledge and assessment, intervention, and referral skills necessary to administer first-tier BSI services.

\section{Role of Internet-Based Intervention}

In the past 10 years, ample research on computer-assisted or Internet-based, automated behavioral interventions has been spurred by possible advantages of consistency, costefficiency, accessibility, convenience, privacy, and anonymity. Although studies suggest that various automated interventions are effective for problem drinking, ${ }^{50-52}$ smoking cessation, ${ }^{53,54}$ and depression, ${ }^{55}$ findings are limited and barriers to dissemination remain.

Dozens of studies of Internet-based, automated alcohol interventions suggest effectiveness, though almost exclusively for college students. ${ }^{50-52}$ In the short term they may be as effective as face-to-face interventions, ${ }^{51}$ but their effectiveness may have been overestimated by faulty statistical analyses. ${ }^{56}$ Findings on long-term effectiveness have been variable. ${ }^{53}$ There is also concern about a substantial gap between efficacy and effectiveness because administration of commercially available programs to large numbers of students has elicited smaller declines in drinking than in earlier research on smaller samples. ${ }^{50}$
Two reviews found inconsistent results among many studies of automated smoking cessation interventions. ${ }^{53,54}$ Frequent automated contacts and strong individual tailoring yielded more effectiveness. Benefits were greater for smokers with more motivation to quit.

Across all behavioral issues, much prior research is flawed. Many automated intervention studies attained poor follow-up response rates. More relevant to dissemination potential, many studies were limited by low participation rates, low program use, and low use of various program components. In many studies, participation rates and effectiveness were especially poor for less educated and older individuals. In one study, program use was enhanced by personal introductions and oversight, ${ }^{55}$ suggesting that a combination of personal and automated interventions might work well for some populations.

Another indication of potential dissemination difficulties came from a study in which few urban general internal medicine patients expressed strong interest in Internet-based programs for a variety of behavioral issues. However, $15 \%$ of patients with no interest in face-to-face assistance did show some interest in Internet-based programs. ${ }^{57}$ Another study showed strong acceptability of an Internet-based intervention by most rural, albeit well-educated, women who are problem drinkers. ${ }^{58}$

Beyond administering automated interventions, the Internet has been used to facilitate interpersonal communication around behavioral issues. In a small study in which therapists provided online feedback on writing assignments, reductions in symptoms of post-traumatic stress disorder were documented up to 18 months later. Another use, without documented effectiveness, has been to assemble synchronous or asynchronous online support groups for depression, cancer, and other chronic diseases. ${ }^{59,60}$

In summary, Internet-administered behavioral interventions hold promise as clinical tools for selected individuals and population niches. Perhaps currently available programs can reduce risky and problem drinking in college student bodies. Otherwise, automated interventions cannot yet be regarded as effective, stand-alone population health management tools.

\section{Conclusion}

The imperatives to improve health outcomes and decrease health care costs require that all primary care settings, emergency departments, and hospitals screen all patients and intervene systematically for tobacco use, excessive drinking, depression, and perhaps other behavioral issues. In most settings, the current staff lack time to provide assessment and intervention services for all patients who would 
screen positive for each behavioral issue. Therefore, most health care teams must be expanded to systematically provide BSI. Receptionists, medical assistants, and nurses could disseminate and review initial health behavior questionnaires. Dedicated, trained, and protocol-guided health educators could serve as frontline BSI providers, conducting assessments with validated questionnaires, delivering interventions, making referrals, and conducting follow-up sessions to gauge progress and need for additional services. Referral resources, including tobacco cessation and alcohol and drug treatment specialists and programs, mental health professionals, dieticians, and exercise coaches, would comprise the second tier of the BSI system. This stepped-care model would conserve current provider and staff time, maximize continuity for patients, and allocate scarce and costly referral resources to those with greatest need and potential to benefit. Perhaps the model could be augmented in the future by various Internet-based resources.

The United States is undergoing an historic, substantial expansion of health care benefits to many more individuals. The proposed stepped-care model to deliver evidence-based BSI systematically would likely improve health outcomes, reduce health care utilization and costs, curtail growth in the need for health care professionals to address acute and chronic illness, enhance workplace productivity, bolster public safety, and defray the costs of expanding health care benefits. Implementation, evaluation, and refinement of the model would be reasonable next steps.

\section{Author Disclosure Statement}

The author is owner and chief executive officer of Wellsys, LLC, which provides consultation, training, support, and software to enable clinical settings to provide systematic behavioral screening and intervention services.

\section{References}

1. Mokdad AH, Marks JS, Stroup DF, Gerberding JL. Actual causes of death in the United States, 2000. JAMA. 2004;291:1238-1245.

2. Centers for Disease Control and Prevention. Chronic diseases and health promotion. Available at http://www .cdc.gov/chronicdisease/overview/index.htm\#2. Accessed November 3, 2010.

3. Centers for Disease Control and Prevention. Tobacco use; targeting the nation's number one killer: At a glance 2010. Available at: http://www.cdc.gov/chronicdisease/resources/ publications/aag/osh.htm. Accessed November 3, 2010.

4. National Institute on Alcohol Abuse and Alcoholism. The economic costs of alcohol abuse. Available at: http://pubs .niaaa.nih.gov/publications/aa51.htm. Accessed November 3, 2010.

5. Office of National Drug Control Policy (ONDCP). The Economic Costs of Drug Abuse in the United States 1992-1998. Pub. No. NCJ-190636. Washington, DC: Executive Office of the President; 2001.

6. Cawley J, Meyerhoefer C. The medical costs of obesity: An instrumental variables approach (NBER Working Paper Number 16467). Available at: http://www.nber.org/papers/ w16467. Accessed November 3, 2010.

7. National Business Group on Health. Moving science into coverage: An employer's guide to preventive services; depression (screening). Available at: http://www.businessgrouphealth. org/preventive/topics/depression.cfm. Accessed November 3, 2010.

8. Lin EHB, Katon W, Von Korrf M, et al. Relationship of depression and diabetes self-care, medication adherence, and preventive care. Diabetes Care. 2004;27:2154-2160.

9. Fiore MC, Baker TB, Bailey WC, et al. Treating tobacco use and dependence: 2008 update. Available at: http://www .ncbi.nlm.nih.gov/bookshelf/br.fcgi?book = hsahcpr\&part $=$ A28163. Accessed November 3, 2010.

10. Kaner EF, Dickinson HO, Beyer FR, et al. Effectiveness of brief alcohol interventions in primary care populations. Cochrane Database of Syst Rev. 2007;(2):CD004148.

11. Lo Sasso AT, Rost K, Beck A. Modeling the impact of enhanced depression treatment on workplace functioning and costs: A cost-benefit approach. Med Care. 2006;44:352-358.

12. Fleming MF, Mundt MP, French MT, Manwell LB, Stauffacher EA, Barry KL. Benefit-cost analysis of brief physician advice with problem drinkers in primary care settings. Med Care. 2000;26:36-43.

13. Gentilello LM, Ebel BE, Wickizer TM, Salkever DS, Rivara FP. Alcohol interventions for trauma patients treated in emergency departments and hospitals: A cost benefit analysis. Ann Surg. 2005;241:541-550.

14. Estee S, Je L, Mancuso D, Felver BEM. Medicaid Costs Declined Among Emergency Department Patients Who Received Brief Interventions for Substance Use Disorders Through WASBIRT. Seattle, WA: Washington State Department of Social \& Health Services; 2007.

15. Madras BK, Compton WM, Avula D, Stegbauer T, Stein JB, Clark HW. Screening, brief interventions, referral to treatment (SBIRT) for illicit drug and alcohol use at multiple healthcare sites: Comparison at intake and 6 months later. Drug Alcohol Depend. 2009;99:280-295.

16. Katon W, Unutzer J, Wells K, Jones L. Collaborative depression care: History, evolution and ways to enhance dissemination and sustainability. Gen Hosp Psychiatry. 2010; 32;456-464.

17. National Institute on Alcohol Abuse and Alcoholism. Brief interventions. Available at: http://pubs.niaaa.nih.gov/publications/aa66/AA66.pdf. Accessed November 3, 2010.

18. Miller WR, Rollnick S. Motivational Interviewing: Preparing People to Change Addictive Behaviors. 2nd ed. New York: Guilford Press; 2002.

19. Larimer ME, Palmer RS, Marlatt GA. Relapse prevention; an overview of Marlatt's cognitive-behavioral model. Alcohol Res Health. 1999;23:151-160.

20. Lejuez CW, Hopko DR, Hopko SD. A brief behavioral activation treatment for depression. Behav Modif. 2001;25: 255-286.

21. Cuijpers P, van Stratten A, Smit F, Mihalapoulous C, Beekman A. Preventing the onset of depressive disorders: A meta-analytic review of psychological interventions. Am J Psychiatry. 2008;165:1272-1280.

22. Sturmey P. Behavioral activation is an evidence-based treatment for depression. Behav Modif. 2009;33:818-829.

23. Dobson KS, Hollon SD, Dimidjian S, et al. Randomized trial of behavioral activation, cognitive therapy, and antidepressant medication in the prevention of relapse and recurrent in major depression. J Consult Clin Psychol. 2008;76:468-477.

24. Fournier JC, DeRubeis JR, Hollon SD, et al. Antidepressant drug effects and depression severity; A patient-level metaanalysis. JAMA. 2010;303:47-53. 
25. US Preventive Services Task Force. USPSTF Grade A and B Recommendations, 2010. Available at: http://www. uspreventiveservicestaskforce.org/uspstf/uspsabrecs.htm. Accessed November 3, 2010.

26. Maciosek MV, Coffield AB, Edwards NM, Goodman MJ, Flottemesch TJ, Solberg LI. Priorities among effective clinical preventive services: Results of a systematic review and analysis. Am J Prev Med. 2006;31:52-61.

27. Stephens RS, Roffman RA, Curtin L. Comparison of extended versus brief treatments for marijuana use. J Consult Clin Psychol. 2000;68:898-908.

28. Copeland J, Swift W, Roffman R, Stephens R. A randomized controlled trial of brief cognitive-behavioral interventions for cannabis use disorder. J Subst Abuse Treat. 2001;21: 55-64.

29. Bernstein J, Bernstein E, Tassiopoulos K, Heeren T, Levenson $\mathrm{S}$, Hingson R. Brief motivational intervention at a clinic visit reduces cocaine and heroin use. Drug Alcohol Depend. 2005;77:49-59.

30. Humeniuk R. The effectiveness of a brief intervention for illicit drugs linked to the alcohol, smoking and substance involvement screening test (ASSIST) in primary health care settings: A technical report of phase III findings of the WHO ASSIST randomized controlled trial. Available at: http:// www.who.int/substance_abuse/activities/assist_technical report_phase3_final.pdf. Accessed November 3, 2010.

31. Bernstein E, Edwards E, Dorfman D, Heeren T, Bliss C, Bernstein J. Screening and brief intervention to reduce marijuana use among youth and young adults in a pediatric emergency department. Acad Emerg Med. 2009;16:11741185.

32. Pomerleau J, Lock K, Knai C, McKee M. Interventions designed to increase adult fruit and vegetable intake can be effective: A systematic review of the literature. J Nutr. 2005;135:2486-2495.

33. Pollak KI, Alexander SC, Coffman CJ, et al. Physician communication techniques and weight loss in adults: Project CHAT. Am J Prev Med. 2010;39:321-328.

34. Foster C, Hillsdon M, Thorogood M. Interventions for promoting physical activity. Cochrane Database Syst Rev. 2005;(1):CD003180.

35. United States Preventive Services Task Force. Behavioral counseling in primary care to promote a healthy diet, 2003. Available at: http://www.uspreventiveservicestaskforce. org/uspstf/uspsdiet.htm. Accessed November 3, 2010.

36. United States Preventive Services Task Force. Behavioral counseling in primary care to promote physical activity, 2002. Available at: http://www.uspreventiveservicestaskforce. org/uspstf/uspsphys.htm. Accessed November 3, 2010.

37. Substance Abuse and Mental Health Services Administration. Results from the 2009 National Survey on Drug Use and Health: Volume I. Summary of National Findings Available at: http://oas.samhsa.gov/NSDUH/2k9NSDUH/ 2k9Results.htm\#Ch4. Accessed November 3, 2010.

38. Substance Abuse and Mental Health Services Administration. Results from the 2008 National Survey on Drug Use and Health: National Findings. Available at: http://oas. samhsa.gov/nsduh/2k8nsduh/2k8Results.cfm\#8.0.4. Accessed November 3, 2010.

39. Centers for Disease Control and Prevention. Behavior Risk Factor Surveillance System; Prevalence and trends data; fruits and vegetables - 2009. Available at: http://apps.nccd.cdc .gov $/$ BRFSS $/$ list.asp $?$ cat $=$ FV\&yr $=2009 \& q k e y=4415 \&$ state $=$ All. Accessed November 3, 2010.
40. Centers for Disease Control and Prevention. Behavior Risk Factor Surveillance System; Prevalence and trends data; physical activity-2009. Available at: http://apps.nccd.cdc.gov/ BRFSS $/$ list.asp $?$ cat $=$ PA\&yr $=2009 \& q k e y=4418 \&$ state $=$ All . Accessed November 3, 2010.

41. Centers for Disease Control and Prevention. Behavior Risk Factor Surveillance System; Prevalence and trends data; overweight and obesity (BMI) - 2009. Available at: http:// apps.nccd.cdc.gov/BRFSS/list.asp?cat $=$ PA\&yr $=2009 \& q k e y=$ 4409\&state $=$ All. Accessed November 3, 2010.

42. American Medical Association. CPT 2010 Professional Edition. Chicago: American Medical Association; 2010.

43. Swanson K, ed. Healthcare Common Procedure Coding System 2010. Los Angeles: Practice Management Information Corporation; 2010.

44. Yarnall KSH, Pollak KI, Ostbye T, Krause KM, Michener JL. Primary care: Is there enough time for prevention? Am J Public Health. 2003;93:635-641.

45. National Committee on Quality Assurance. Standards and guidelines for Physician Practice Connections-Patient-Centered Medical Home, 2007. Available at: http://www.ncqa.org/ tabid/631/default.aspx. Accessed November 3, 2010.

46. Glasgow RE, Orleans CT, Wagner EH. Does the chronic care model also serve as a template for improving prevention? Milbank Q. 2001;79:579-612.

47. Hettema J, Steele J, Miller WR. Motivational interviewing. Ann Rev Clin Pychol. 2005;1:91-111.

48. National Commission for Health Education Credentialing. Responsibilities and competencies of health educators. Available at: http://www.nchec.org/credentialing/responsibilities/. Accessed November 3, 2010.

49. Holtrop JS, Jordan TR. The patient-centered home and why it matters to health educators. Health Promot Pract. 2010;11:622-628.

50. Carey KB, Scott-Sheldon LAJ, Elliott JC, Bolles JR, Carey MP. Computer-delivered interventions to reduce college student drinking: A meta-analysis. Addiction. 2009;104:1807-1819.

51. Tait RJ, Christensen H. Internet-based interventions for young people with problematic substance use: A systematic review. Med J Aust. 2010;192(11 suppl):S15-S21.

52. Vernon ML. A review of computer-based alcohol problem services designed for the general public. J Subst Abuse Treat. 2010;38:203-211.

53. Civljak M, Sheikh A, Stead LF, Car J. Internet-based interventions for smoking cessation. Cochrane Database Syst Rev. 2010;(9):CD007078.

54. Shahab L, McEwen A. Online support for smoking cessation: A systematic review of the literature. Addiction. 2009; 104:1792-1804.

55. Levin W, Campbell DR, McGovern KB, et al. A computerassisted depression intervention in primary care. Psychol Med. Epub ahead of print 20 October 2010 doi:10.1017/ S0033291710001935.

56. Khadjesari Z, Murray E, Hewitt C, Hartley S, Godfrey C. Can stand-alone computer-based interventions reduce alcohol consumption? A systematic review. Addiction. 2011; 106:267-282.

57. Mohr DC, Siddique J, Ho J, Duffecy J, Jin L, Fokuo JK. Interest in behavioral and psychological treatments delivered face-to-face, by telephone, and by Internet. Ann Behav Med. 2010;40:89-98.

58. Finfgeld-Connett D. Web-based treatment for rural women with alcohol problems; Preliminary findings. Comput Inform Nurs. 2009;27:345-353. 
59. Griffiths KM, Calear AL, Banfield M, Tam A. Systematic review on Internet support groups (ISGs) for depression: What is known about depression ISGs? J Med Internet Res. 2009;11:e41.

60. Griffiths KM, Calear AL, Banfield M. Systematic review on internet support groups (ISGs) for depression: Do ISGs reduce depressive symptoms? J Med Internet Res. 2009; 11:e40.
Address correspondence to: Richard L. Brown, MD, MPH

Department of Family Medicine School of Medicine and Public Health University of Wisconsin Madison, WI

E-mail: rlbrown@wisc.edu 

Copyright of Population Health Management is the property of Mary Ann Liebert, Inc. and its content may not be copied or emailed to multiple sites or posted to a listserv without the copyright holder's express written permission. However, users may print, download, or email articles for individual use. 